\title{
Global stability of SIRS epidemic models with a class of nonlinear incidence rates and distributed delays
}

\author{
Yoichi Enatsu $^{\mathrm{a}, *}$, Yukihiko Nakata ${ }^{\mathrm{b}}$, Yoshiaki Muroya $^{\mathrm{c}}$ \\ ${ }^{a}$ Department of Pure and Applied Mathematics, Waseda University, 3-4-1 Ohkubo, \\ Shinjuku-ku, Tokyo, 169-8555, Japan \\ ${ }^{b}$ Basque Center for Applied Mathematics, Bizkaia Technology Park, Building 500 E-48160 \\ Derio, Spain \\ ${ }^{c}$ Department of Mathematics, Waseda University 3-4-1 Ohkubo, Shinjuku-ku, Tokyo \\ 169-8555, Japan
}

\begin{abstract}
In this paper, we establish the global asymptotic stability of a disease-free equilibrium and an endemic equilibrium of an SIRS epidemic model with a class of nonlinear incidence rates and distributed delays. By using strict monotonicity of the incidence function and constructing a Lyapunov functional, we obtain sufficient conditions under which the endemic equilibrium is globally asymptotically stable. When the nonlinear incidence rate is a saturated incidence rate, our result provides a new global stability condition for a small rate of immunity loss.
\end{abstract}

Keywords: SIRS epidemic model; nonlinear incidence rate; global asymptotic stability; distributed delays; Lyapunov functional 2000 MSC: 34K20, 34K25, 92D30.

\section{Introduction}

Mathematical models which describe the dynamics of infectious diseases have played a crucial role in the disease control in epidemiological aspects. In order to understand the mechanism of disease transmission, many authors have proposed various kinds of epidemic models (see [1]-[19] and the references therein).

Much attention has been paid to the analysis of the stability of the diseasefree equilibrium and the endemic equilibrium of the epidemic models. MenaLorca and Hethcote [15] considered several SIRS epidemic models with a bilinear incidence rate which takes the form $\beta S(t) I(t)$ and a standard incidence rate which takes the form $\beta S(t) I(t) / N(t)$, where $N(t)=S(t)+I(t)+R(t)$. A threshold parameter of the models was also found in Mena-Lorca and Hethcote

\footnotetext{
* Corresponding author.

Email addresses: yo1.gc-rw.docomo@akane.waseda.jp (Yoichi Enatsu), nakata@bcamath.org (Yukihiko Nakata), ymuroya@waseda.jp (Yoshiaki Muroya)
} 
[15] to determine whether the disease dies out or approaches to an endemic equilibrium. Later, in order to investigate the effect of an immunity loss of diseases, various kinds of SIRS epidemic models and a significant body of work concerning the stability analysis of the steady states of the models have been carried out (see, for example, [7, 8, 9, 10, 11, 12, 16, 17, 19] and the references therein).

In modeling of those communicable diseases, an incidence rate has played a vital role in ensuring that the model can give a reasonable qualitative description for the disease dynamics. A bilinear incidence rate and a standard incidence rate were frequently used in the literature of mathematical modeling. On the other hand, many authors have suggested that transmission of the infection shall have a nonlinear incidence rate. For example, Capasso and Serio [2] studied the cholera epidemic spread in Bari in 1973 and have given an assumption that the incidence rate takes the nonlinear form $\frac{\beta S(t) I(t)}{1+\alpha I(t)}$, which has been interpreted as a saturated incidence rate. The saturation effect has been originally introduced for the Holling functional response of the predator in a prey-predator system. This incidence rate includes the behavioral change and crowding effect of the infective individuals and prevents the unboundedness of the contact rate. Korobeinikov and Maini [9] thereafter formulated a variety of models with an incidence rate of the form $F(S(t)) G(I(t))$ and Korobeinikov $[10,11]$ obtained the global properties of basic SIR and SIRS epidemic models with a more general framework of the incidence rate $F(S(t), I(t))$ but no delays.

Recently, Xu and Ma [18] investigated the spread of an infectious disease transmitted by a vector (e.g. mosquitoes, rats, etc.) after an incubation time $\tau$ denoting the time during which the infectious agents develop in the vector, that is, they incorporated time delay effects and formulated an SIRS epidemic model with the saturated incidence rate $\beta S(t) \frac{I(t-h)}{1+\alpha I(t-h)}$ (see also Section 6). More authors have now proposed several reasons for the nonlinearity of the incidence rates and introduced various nonlinear incidence functions with delays (see also $[7,19])$.

In this paper, in order to study the impact of nonlinearity of those incidence rates and time delay effects, we consider the global dynamics of the following SIRS epidemic model with a class of nonlinear incidence rates and distributed delays:

$$
\left\{\begin{aligned}
\frac{\mathrm{d} S(t)}{\mathrm{d} t} & =B-\mu S(t)-\beta S(t) \int_{0}^{h} f(\tau) G(I(t-\tau)) \mathrm{d} \tau+\delta R(t), \\
\frac{\mathrm{d} I(t)}{\mathrm{d} t} & =\beta S(t) \int_{0}^{h} f(\tau) G(I(t-\tau)) \mathrm{d} \tau-(\mu+\gamma) I(t), \\
\frac{\mathrm{d} R(t)}{\mathrm{d} t} & =\gamma I(t)-(\mu+\delta) R(t) .
\end{aligned}\right.
$$

$S(t), I(t)$ and $R(t)$ denote the numbers of susceptible, infective and recovered individuals at time $t$, respectively. $B>0$ is the recruitment rate of the population, $\mu>0$ is the natural death rate of the population, $\beta>0$ is the 
proportionality constant, $\gamma>0$ is the natural recovery rate of the infective individuals, $\delta \geq 0$ is the rate at which recovered individuals lose immunity and return to the susceptible class. $h>0$ is a maximum time taken to become infectious and the transmission of the infection is governed by an incidence rate $\beta S(t) \int_{0}^{h} f(\tau) G(I(t-\tau)) \mathrm{d} \tau . \quad f(\tau)$ denotes the fraction of vector population in which the time taken to become infectious is $\tau$. Here, $f:[0, h] \rightarrow[0,+\infty)$ is continuous on $[0, h]$ satisfying $\int_{0}^{h} f(\tau) \mathrm{d} \tau=1$. The initial conditions for system (1.1) take the form

$$
S(\theta)=\varphi_{1}(\theta), \quad I(\theta)=\varphi_{2}(\theta), \quad R(\theta)=\varphi_{3}(\theta), \quad-h \leq \theta \leq 0,
$$

where $\varphi=\left(\varphi_{1}, \varphi_{2}, \varphi_{3}\right)^{T} \in C$ such that $\varphi_{i}(\theta)=\varphi_{i}(0) \geq 0(-h \leq \theta \leq 0, i=$ $1,3), \varphi_{2}(\theta) \geq 0(-h \leq \theta \leq 0)$. $C$ denotes the Banach space $C\left([-h, 0], \mathbb{R}_{+0}^{3}\right)$ of continuous functions mapping the interval $[-h, 0]$ into $\mathbb{R}_{+0}^{3}$ with the supremum norm, where $\mathbb{R}_{+0}^{n}=\left\{\left(x_{1}, \cdots, x_{n}\right) \mid x_{i} \geq 0, i=1, \cdots, n\right\}$ for $n \geq 1$. From a biological meaning, we assume that $\varphi_{i}(0)>0$ for $i=1,2,3$.

Throughout the paper, we further assume that

(H1) $G(I)$ is continuous and monotone increasing on $[0,+\infty)$ with $G(0)=0$.

(H2) $I / G(I)$ is monotone increasing on $(0,+\infty)$ with $\lim _{I \rightarrow+0}(I / G(I))=1$.

We here note that $G$ is a Lipschitz continuous function on $[0,+\infty)$.

We define the basic reproduction number of system (1.1) by

$$
R_{0}=\frac{B \beta}{\mu(\mu+\gamma)} .
$$

$R_{0}$ denotes the expected number of secondary infectious cases generated by one typical primary case in an entirely susceptible and sufficiently large population.

It is well known by the fundamental theory of functional differential equations that the solution $(S(t), I(t), R(t))$ of system (1.1) is unique and positive for all $t \geq 0$. System (1.1) always has a disease-free equilibrium $E_{0}=(B / \mu, 0,0)$ and if $R_{0}>1$, then system (1.1) allows a unique endemic equilibrium $E_{*}=$ $\left(S^{*}, I^{*}, R^{*}\right), S^{*}>0, I^{*}>0$ and $R^{*}>0$ (see Lemma 4.1).

In the present paper, applying a Lyapunov functional technique for a delayed SIR epidemic model in McCluskey [13] and the property that the total population $N(t)$ of system (1.1) converges to a positive constant $B / \mu$, we obtain a sufficient condition which ensures the global asymptotic stability of the endemic equilibrium $E_{*}$ of system (1.1) for $R_{0}>1$ (see Lemma 5.1). The main results are as follows.

Theorem 1.1 If $R_{0}<1$, then the disease-free equilibrium $E_{0}$ of system (1.1) is globally asymptotically stable.

Theorem 1.2 If $R_{0}>1$, then the endemic equilibrium $E_{*}$ of system (1.1) exists uniquely and system (1.1) is permanent. Moreover, assume that the following conditions hold. 
(I) There exist positive constants $C_{1}$ and $C_{2}$ such that

$$
\begin{aligned}
& \inf _{0 \leq I \leq B / \mu} \frac{G(I)-G\left(I^{*}\right)}{I-I^{*}} \geq C_{1}>0 \text { and } \inf _{0<I \leq B / \mu} \frac{\frac{I}{G(I)}-\frac{I^{*}}{G\left(I^{*}\right)}}{I-I^{*}} \geq C_{2}>0, \\
& \text { (II) } \delta^{2}-4 C_{1} C_{2}(\mu+\gamma)(\mu+\delta) \frac{B}{\mu+\beta G(B / \mu)}<0 .
\end{aligned}
$$

Then the endemic equilibrium $E_{*}$ of system (1.1) is globally asymptotically stable.

We show the global asymptotic stability of the endemic equilibrium for a small rate of immunity loss $\delta$ as long as the infection rate has suitable properties concerning the concavity of function $G(I)$ characterized by the hypotheses $(\mathrm{H} 1)$ and (H2).

The organization of this paper is the following. In Section 2, we offer a basic result for system (1.1). In Section 3, we prove Theorem 1.1. In Section 4 , we establish the permanence and global asymptotic stability of the endemic equilibrium $E_{*}$ of system (1.1) for $R_{0}>1$. In Section 5 , we prove Theorem 1.2 by means of Lyapunov functionals on a reduced system which is derived from system (1.1) with a key lemma (see Lemma 5.1). To show the feasibility of our global stability conditions of the endemic equilibrium for $R_{0}>1$, we offer numerical examples in Section 6. Finally, a discussion is offered in Section 7.

\section{A basic result}

We now state a basic result of system (1.1). The following lemma will be used in the proofs of Theorem 1.1, Theorem 1.2 and Lemma 4.2.

Lemma 2.1 The plane $S(t)+I(t)+R(t)=B / \mu$ is an invariant manifold of system (1.1), which is globally attractive in the first octant of $\mathbb{R}^{3}$, that is,

$$
\lim _{t \rightarrow+\infty}(S(t)+I(t)+R(t))=\frac{B}{\mu} .
$$

Proof. Let $N(t)=S(t)+I(t)+R(t)$. Then it follows from system (1.1) that

$$
\begin{aligned}
\frac{\mathrm{d} N(t)}{\mathrm{d} t} & =B-\mu S(t)-\mu I(t)-\mu R(t) \\
& =B-\mu N(t) .
\end{aligned}
$$

Hence, we obtain that $\lim _{t \rightarrow+\infty} N(t)=B / \mu$. This completes the proof.

\section{Stability of the disease-free equilibrium $E_{0}$ for $R_{0}<1$}

\subsection{Local stability of the disease-free equilibrium $E_{0}$}

We now give the following theorem concerning the local asymptotic stability of the disease-free equilibrium $E_{0}$ of system (1.1). 
Lemma 3.1 If $R_{0}<1$, then the disease free equilibrium $E_{0}$ of system (1.1) is locally asymptotically stable. Furthermore, the disease-free equilibrium $E_{0}$ of system (1.1) is unstable if $R_{0}>1$.

Proof. By the hypothesis (H2), the characteristic equation of system (1.1) at the disease-free equilibrium $E_{0}$ is of the form

$$
(\lambda+\mu)\left\{\lambda+(\mu+\gamma)\left(1-R_{0} \int_{0}^{h} f(\tau) \mathrm{e}^{-\lambda \tau} \mathrm{d} \tau\right)\right\}(\lambda+\mu+\delta)=0 .
$$

Clearly, $\lambda=-\mu,-(\mu+\delta)$ are always roots of (3.1). All other roots of (3.1) are determined by the following equation:

$$
\lambda+(\mu+\gamma)\left(1-R_{0} \int_{0}^{h} f(\tau) \mathrm{e}^{-\lambda \tau} \mathrm{d} \tau\right)=0 .
$$

For the case $R_{0}<1$, we suppose to the contrary that the disease-free equilibrium $E_{0}$ is not locally asymptotically stable. Then, there exists a root $\lambda=\tilde{\lambda}$ such that $\operatorname{Re} \tilde{\lambda} \geq 0$. However, from (3.2), we obtain

$$
\begin{aligned}
\operatorname{Re} \tilde{\lambda} & =(\mu+\gamma)\left\{R_{0} \exp (-\operatorname{Re} \tilde{\lambda} \tau) \int_{0}^{h} f(\tau) \cos (\operatorname{Im} \tilde{\lambda} \tau) d \tau-1\right\} \\
& \leq(\mu+\gamma)\left(R_{0}-1\right)<0,
\end{aligned}
$$

which is a contradiction. Hence, if $R_{0}<1$, then the disease-free equilibrium $E_{0}$ of system (1.1) is locally asymptotically stable. Now, we put

$$
T(\lambda):=\lambda+(\mu+\gamma)\left(1-R_{0} \int_{0}^{h} f(\tau) \mathrm{e}^{-\lambda \tau} \mathrm{d} \tau\right) .
$$

For the case $R_{0}>1$, it is directly seen from (3.3) that, for $\lambda \in \mathbb{R}$,

$$
T(0)=(\mu+\gamma)\left(1-R_{0}\right)<0, \lim _{\lambda \rightarrow+\infty} T(\lambda)=+\infty .
$$

Therefore, (3.1) has at least one positive real root. Hence, if $R_{0}>1$, then the disease-free equilibrium $E_{0}$ is unstable. This completes the proof.

\subsection{Global stability of the disease-free equilibrium $E_{0}$}

In this subsection, by constructing a Lyapunov functional, we prove Theorem 1.1.

Proof of Theorem 1.1. First, by (1.3) and $R_{0}<1$, we choose $\varepsilon_{s}>0$ sufficiently small such that

$$
\beta\left(\frac{B}{\mu}+\varepsilon_{s}\right)<\mu+\gamma .
$$


We consider the following Lyapunov functional:

$$
W(t)=I(t)+(\mu+\gamma) \int_{0}^{h} f(\tau) \int_{t-\tau}^{t} I(u) \mathrm{d} u \mathrm{~d} \tau .
$$

From Lemma 2.1, one can see that for $\varepsilon_{s}>0$, there is a $T>0$ such that $S(t) \leq \frac{B}{\mu}+\varepsilon_{s}$, for $t>T$. Then we obtain

$$
\begin{aligned}
\frac{\mathrm{d} W(t)}{\mathrm{d} t}= & \beta S(t) \int_{0}^{h} f(\tau) G(I(t-\tau)) \mathrm{d} \tau-(\mu+\gamma) I(t) \\
& \quad+(\mu+\gamma) \int_{0}^{h} f(\tau)(I(t)-I(t-\tau)) \mathrm{d} \tau \\
\leq & \beta\left(\frac{B}{\mu}+\varepsilon_{s}\right) \int_{0}^{h} f(\tau) G(I(t-\tau)) \mathrm{d} \tau-(\mu+\gamma) \int_{0}^{h} f(\tau) I(t-\tau) \mathrm{d} \tau \\
= & \beta\left(\frac{B}{\mu}+\varepsilon_{s}\right) \int_{0}^{h} f(\tau) I(t-\tau) \mathrm{d} \tau-(\mu+\gamma) \int_{0}^{h} f(\tau) I(t-\tau) \mathrm{d} \tau \\
= & \int_{0}^{h} f(\tau)\left\{\beta\left(\frac{B}{\mu}+\varepsilon_{s}\right)-(\mu+\gamma)\right\} I(t-\tau) \mathrm{d} \tau,
\end{aligned}
$$

for $t>T+h$. From (3.4) and arbitrarity of $\varepsilon_{s}>0$, we obtain that $\frac{\mathrm{d} W(t)}{\mathrm{d} t} \leq 0$ holds for $t>T+h$. Thus, it holds that $\lim _{t \rightarrow+\infty} W(t)=0$, which implies that $\lim _{t \rightarrow+\infty} I(t)=0$. It follows that $\lim _{t \rightarrow+\infty} R(t)=0$ and $\lim _{t \rightarrow+\infty} S(t)=B / \mu$ hold. By Lemma 3.1 and Lyapunov-LaSalle asymptotic stability theorem, the disease-free equilibrium $E_{0}$ of system (1.1) is globally asymptotically stable. This completes the proof.

Remark 3.1 To establish the global asymptotic stability of the disease-free equilibrium $E_{0}$ of system (1.1) for $R_{0}<1$, the hypothesis of the monotonicity of $G(I)$ of $I \geq 0$ in (H1) is not necessary for our analysis.

\section{Permanence for $R_{0}>1$}

4.1. Existence and uniqueness of the endemic equilibrium $E_{*}$

In this subsection, by the hypothesis (H2), we give a lemma of the unique existence of the endemic equilibrium $E_{*}$ of system (1.1) for $R_{0}>1$ :

Lemma 4.1 If $R_{0}>1$, then system (1.1) has a unique endemic equilibrium $E_{*}$ satisfying

$$
\left\{\begin{array}{l}
B-\mu S^{*}-\beta S^{*} G\left(I^{*}\right)+\delta R^{*}=0 \\
\beta S^{*} G\left(I^{*}\right)-(\mu+\gamma) I^{*}=0 \\
\gamma I^{*}-(\mu+\delta) R^{*}=0
\end{array}\right.
$$


Proof. Assume that $R_{0}>1$. From the second and the third equations of (4.1), it holds that

$$
S^{*}=\frac{(\mu+\gamma) I^{*}}{\beta G\left(I^{*}\right)}
$$

and

$$
R^{*}=\frac{\gamma I^{*}}{\mu+\delta}
$$

After substituting (4.2) into the first equation of (4.1), we consider the following equation:

$$
H(I):=B-\frac{\mu(\mu+\gamma) I}{\beta G(I)}-\frac{\mu(\mu+\gamma+\delta)}{\mu+\delta} I=0 .
$$

By the hypothesis (H2), one can see that $H(I)$ is a strictly monotone decreasing function of $I \in(0,+\infty)$ satisfying

$$
\lim _{I \rightarrow+0} H(I)=B-\frac{\mu(\mu+\gamma)}{\beta}=B\left(1-\frac{1}{R_{0}}\right)>0
$$

and $H(I)<0$ holds for any $I>\frac{B(\mu+\delta)}{\mu(\mu+\gamma+\delta)}$. Hence, there exists a unique positive $I^{*}>0$ such that $H\left(I^{*}\right)=0$. By (4.2) and (4.3), there exists a unique endemic equilibrium $E_{*}=\left(S^{*}, I^{*}, R^{*}\right)$. Hence, the proof is complete.

\subsection{Permanence for $R_{0}>1$}

In this subsection, we show the permanence of system (1.1). The following lemma indicates that the disease eventually persists in the host population if $R_{0}>1$.

Lemma 4.2 If $R_{0}>1$, then for any solutions of system (1.1), it holds that

$$
\left\{\begin{array}{l}
\liminf _{t \rightarrow+\infty} S(t) \geq v_{1}:=\frac{B}{\mu+\beta G(B / \mu)}, \\
\liminf _{t \rightarrow+\infty} I(t) \geq v_{2}:=q I^{*} \mathrm{e}^{-(\mu+\gamma)(h+\rho h)}, \\
\liminf _{t \rightarrow+\infty} R(t) \geq v_{3}:=\frac{\gamma v_{2}}{\mu+\delta},
\end{array}\right.
$$

where $q>0$ and $\rho>0$ satisfy $S^{*}<S^{\triangle}:=\frac{B}{k}\left(1-\mathrm{e}^{-k \rho h}\right), k=\mu+\beta G\left(q I^{*}\right)$.

Proof. Let $(S(t), I(t), R(t))$ be a solution of system (1.1) with initial condition (1.2). By Lemma 2.1, it holds that $\lim \sup _{t \rightarrow+\infty} I(t) \leq \frac{B}{\mu}$. For $\varepsilon_{I}>0$ sufficiently small, there is a $T_{1}>0$ such that $I(t) \leq \frac{B}{\mu}+\varepsilon_{I}$ for $t>T_{1}$. From the hypothesis (H1), we therefore derive from the first equation of system (1.1)

$$
\frac{\mathrm{d} S(t)}{\mathrm{d} t} \geq B-\left\{\mu+\beta G\left(\frac{B}{\mu}+\varepsilon_{I}\right)\right\} S(t),
$$


which implies that

$$
\liminf _{t \rightarrow+\infty} S(t) \geq \frac{B}{\mu+\beta G\left(B / \mu+\varepsilon_{I}\right)} .
$$

Since the above inequality holds for arbitrary $\varepsilon_{I}>0$ sufficiently small, it follows that $\liminf _{t \rightarrow+\infty} S(t) \geq v_{1}$.

We now show that $\liminf _{t \rightarrow+\infty} I(t) \geq v_{2}$. First, we prove that it is impossible that $I(t) \leq q I^{*}$ for all $t \geq \rho h$. Suppose to the contrary that $I(t) \leq q I^{*}$ for all $t \geq \rho h$. From the first equation of system (1.1), one can obtain that

$$
\frac{\mathrm{d} S(t)}{\mathrm{d} t} \geq B-\left(\mu+\beta G\left(q I^{*}\right)\right) S(t), \text { for } t \geq \rho h+h,
$$

which yields

$$
\begin{aligned}
S(t) & \geq \mathrm{e}^{-k(t-\rho h-h)}\left\{S(\rho h+h)+B \int_{\rho h+h}^{t} \mathrm{e}^{k(\theta-\rho h-h)} \mathrm{d} \theta\right\} \\
& >\frac{B}{k}\left(1-\mathrm{e}^{-k(t-\rho h-h)}\right), \text { for } t \geq \rho h+h .
\end{aligned}
$$

Hence it follows from (4.4) that

$$
S(t)>\frac{B}{k}\left(1-\mathrm{e}^{-k \rho h}\right)=S^{\triangle}>S^{*}, \text { for } t \geq 2 \rho h+h .
$$

For $t \geq 0$, we define

$$
V(t)=I(t)+\beta S^{*} \int_{0}^{h} f(\tau) \int_{t-\tau}^{t} G(I(u)) \mathrm{d} u \mathrm{~d} \tau .
$$

Noting that $I(t) \leq q I^{*}<I^{*}$, calculating the derivative of $V(t)$ along solutions of system (1.1) gives as follows.

$$
\begin{aligned}
& \frac{\mathrm{d} V(t)}{\mathrm{d} t} \\
= & \beta \int_{0}^{h} f(\tau) G(I(t-\tau))\left(S(t)-S^{*}\right) \mathrm{d} \tau+\beta S^{*} G(I(t))-(\mu+\gamma) I(t) \\
= & \beta \int_{0}^{h} f(\tau) G(I(t-\tau))\left(S(t)-S^{*}\right) \mathrm{d} \tau+\left\{\beta S^{*} \frac{G(I(t))}{I(t)}-(\mu+\gamma)\right\} I(t) \\
\geq & \beta \int_{0}^{h} f(\tau) G(I(t-\tau))\left(S(t)-S^{*}\right) \mathrm{d} \tau+\left\{\beta S^{*} \frac{G\left(I^{*}\right)}{I^{*}}-(\mu+\gamma)\right\} I(t) \\
= & \beta \int_{0}^{h} f(\tau) G(I(t-\tau))\left(S(t)-S^{*}\right) \mathrm{d} \tau \\
> & \beta \int_{0}^{h} f(\tau) G(I(t-\tau))\left(S^{\triangle}-S^{*}\right) \mathrm{d} \tau, \text { for } t \geq 2 \rho h+h .
\end{aligned}
$$


Setting $\underline{i}=\min _{\theta \in[-h, 0]} I(\theta+2 \rho h+2 h)$, we claim that $I(t) \geq \underline{i}$ for all $t \geq 2 \rho h+h$. Otherwise, if there is a $T \geq 0$ such that $I(t) \geq \underline{i}$ for $2 \rho h+h \leq t \leq 2 \rho h+2 h+T$, $I(2 \rho h+2 h+T)=\underline{i}$ and $\left.\frac{\mathrm{d}}{\mathrm{d} t} I(t)\right|_{t=2 \rho h+2 h+T} \leq 0$, then it follows from (4.5), the second equation of (4.1) that

$$
\begin{aligned}
\left.\frac{\mathrm{d} I(t)}{\mathrm{d} t}\right|_{t=2 \rho h+2 h+T} & =\beta S(t) \int_{0}^{h} f(\tau) G(I(t-\tau)) \mathrm{d} \tau-(\mu+\gamma) I(t) \\
& \geq \beta S(t) G(I(t))-(\mu+\gamma) I(t) \\
& \geq\left\{\beta S(t) \frac{G\left(I^{*}\right)}{I^{*}}-(\mu+\gamma)\right\} \underline{i} \\
& >\left\{\beta S^{\triangle} \frac{G\left(I^{*}\right)}{I^{*}}-(\mu+\gamma)\right\} \underline{i} \\
& >\left\{\beta S^{*} \frac{G\left(I^{*}\right)}{I^{*}}-(\mu+\gamma)\right\} \underline{i}=0 .
\end{aligned}
$$

This is a contradiction. Therefore $I(t) \geq \underline{i}$ for all $t \geq 2 \rho h+h$. By the hypothesis (H1), it follows from (4.7) that

$$
\frac{\mathrm{d} V(t)}{\mathrm{d} t}>\beta G(\underline{i})\left(S^{\triangle}-S^{*}\right)>0, \text { for } t \geq 2 \rho h+2 h,
$$

which implies that $\lim _{t \rightarrow+\infty} V(t)=+\infty$. However, from Lemma 2.1, it holds that $\limsup _{t \rightarrow+\infty} V(t) \leq \frac{B}{\mu}+\beta S^{*} G\left(\frac{B}{\mu}\right)<+\infty$. This leads to a contradiction. Hence the claim is proved.

Second, since the above claim holds, we are left to consider two possibilities:

$\left\{\right.$ (i) $I(t) \geq q I^{*}$ for all $t$ sufficiently large,

(ii) $I(t)$ oscillates about $q I^{*}$ for all $t$ sufficiently large.

If the first case holds, then we immediately get the conclusion. If the second case holds, we show that $I(t) \geq v_{2}$ for all $t$ sufficiently large. Let $t_{1}<t_{2}$ be sufficiently large such that

$$
I\left(t_{1}\right)=I\left(t_{2}\right)=q I^{*}, I(t)<q I^{*}, t_{1}<t<t_{2} .
$$

If $t_{2}-t_{1} \leq h+\rho h$, then it follows from the second equation of system (1.1) that

$$
\frac{\mathrm{d} I(t)}{\mathrm{d} t} \geq-(\mu+\gamma) I(t)
$$

that is,

$$
I(t) \geq I\left(t_{1}\right) \mathrm{e}^{-(\mu+\gamma)\left(t-t_{1}\right)}=q I^{*} \mathrm{e}^{-(\mu+\gamma)(h+\rho h)}=v_{2} .
$$

If $t_{2}-t_{1}>h+\rho h$, we similarly obtain from the second equation of system (1.1) that $I(t) \geq v_{2}$ for $t_{1} \leq t \leq t_{1}+h+\rho h$. We now claim that $I(t) \geq v_{2}$ for all $t_{1}+h+\rho h \leq t \leq t_{2}$. Otherwise, there is a $T^{*}>0$ such that $I(t) \geq v_{2}$ for 
$t_{1} \leq t \leq t_{1}+h+\rho h+T^{*}, I\left(t_{1}+h+\rho h+T^{*}\right)=v_{2}$ and $\left.\frac{\mathrm{d} I(t)}{\mathrm{d} t}\right|_{t=t_{1}+h+\rho h+T^{*}} \leq 0$. On the other hand, it follows from the second equation of system (1.1) that

$$
\begin{aligned}
\left.\frac{\mathrm{d} I(t)}{\mathrm{d} t}\right|_{t=t_{1}+h+\rho h+T^{*}} & =\beta S(t) \int_{0}^{h} f(\tau) G(I(t-\tau)) \mathrm{d} \tau-(\mu+\gamma) I(t) \\
& \geq \beta S^{\triangle} G(I(t))-(\mu+\gamma) I(t) \\
& \geq\left\{\beta S^{\triangle}-(\mu+\gamma) \frac{v_{2}}{G\left(v_{2}\right)}\right\} G\left(v_{2}\right) .
\end{aligned}
$$

By (4.1), (4.5) and the hypothesis (H2), it holds that

$$
\left.\frac{\mathrm{d} I(t)}{\mathrm{d} t}\right|_{t=t_{1}+h+\rho h+T^{*}} \geq\left\{\beta S^{\triangle}-(\mu+\gamma) \frac{I^{*}}{G\left(I^{*}\right)}\right\} G\left(v_{2}\right)>0,
$$

which is a contradiction. Hence $I(t) \geq v_{2}$ for $t_{1} \leq t \leq t_{2}$. Since the interval $\left[t_{1}, t_{2}\right]$ is arbitrarily chosen, we conclude that $I(t) \geq v_{2}$ holds for all $t$ sufficiently large for the second case. Thus, we obtain $\liminf _{t \rightarrow+\infty} I(t) \geq v_{2}$, from which we have $\liminf \operatorname{int}_{t \rightarrow \infty} R(t) \geq v_{3}$. Hence, this completes the proof.

\section{Global stability of the endemic equilibrium $E_{*}$ for $R_{0}>1$}

From Lemma 2.1, we see that the limit set of system (1.1) in the first octant of $\mathbb{R}^{3}$ locates on the plane $S+I+R=B / \mu$. Hence, the dynamics of system (1.1) in the first octant of $\mathbb{R}^{3}$ is equivalent to the following system:

$$
\left\{\begin{array}{l}
\frac{\mathrm{d} S(t)}{\mathrm{d} t}=\frac{B(\mu+\delta)}{\mu}-(\mu+\delta) S(t)-\beta S(t) \int_{0}^{h} f(\tau) G(I(t-\tau)) \mathrm{d} \tau-\delta I(t), \\
\frac{\mathrm{d} I(t)}{\mathrm{d} t}=\beta S(t) \int_{0}^{h} f(\tau) G(I(t-\tau)) \mathrm{d} \tau-(\mu+\gamma) I(t) .
\end{array}\right.
$$

We now discuss the global asymptotic stability of the endemic equilibrium $\tilde{E}_{*}$ of system (5.1) for $R_{0}>1$. By Lemma 4.1, we recall that the existence of the endemic equilibrium $\tilde{E}_{*}:=\left(S^{*}, I^{*}\right)$ of system (5.1) is guaranteed for the case $R_{0}>1$. Put

$$
x_{t}=\frac{S(t)}{S^{*}}, y_{t}=\frac{I(t)}{I^{*}}, \tilde{y}_{t}=\frac{G(I(t))}{G\left(I^{*}\right)} .
$$

The following key lemma plays an important role to obtain Theorem 1.2.

Lemma 5.1 For all $t \geq 0$, under the condition (I), it holds that

$$
g\left(y_{t}\right)-g\left(\tilde{y}_{t}\right) \geq C_{1} C_{2} I^{*}\left(y_{t}-1\right)^{2},
$$

where $g(x)=x-1-\ln x \geq g(1)=0$ defined for all $x>0$. 
Proof. First, we obtain that

$$
\tilde{y}_{t}-1=\frac{G(I(t))-G\left(I^{*}\right)}{G\left(I^{*}\right)}
$$

and

$$
y_{t}-\tilde{y}_{t}=\frac{I(t)}{I^{*}}-\frac{G(I(t))}{G\left(I^{*}\right)}=\frac{G(I(t))}{I^{*}}\left(\frac{I(t)}{G(I(t))}-\frac{I^{*}}{G\left(I^{*}\right)}\right) .
$$

Then it follows from the condition (I) that

$$
\begin{aligned}
\left(\tilde{y}_{t}-1\right)\left(y_{t}-\tilde{y}_{t}\right) & =\frac{G(I(t))}{I^{*} G\left(I^{*}\right)}\left(G(I(t))-G\left(I^{*}\right)\right)\left(\frac{I(t)}{G(I(t))}-\frac{I^{*}}{G\left(I^{*}\right)}\right) \\
& \geq \frac{C_{1} C_{2} G(I(t))}{I^{*} G\left(I^{*}\right)}\left(I(t)-I^{*}\right)^{2} \\
& =\frac{C_{1} C_{2} I^{*} G(I(t))}{G\left(I^{*}\right)}\left(y_{t}-1\right)^{2} \\
& =C_{1} C_{2} I^{*} \tilde{y}_{t}\left(y_{t}-1\right)^{2} .
\end{aligned}
$$

By $g^{\prime \prime}(x)=\frac{1}{x^{2}}>0$ for $x>0$ and (5.4), we obtain that

$$
\begin{aligned}
g\left(y_{t}\right)-g\left(\tilde{y}_{t}\right) & \geq g^{\prime}\left(\tilde{y}_{t}\right)\left(y_{t}-\tilde{y}_{t}\right) \\
& =\left(1-\frac{1}{\tilde{y}_{t}}\right)\left(y_{t}-\tilde{y}_{t}\right) \\
& =\frac{1}{\tilde{y}_{t}}\left(\tilde{y}_{t}-1\right)\left(y_{t}-\tilde{y}_{t}\right) \\
& \geq C_{1} C_{2} I^{*}\left(y_{t}-1\right)^{2} .
\end{aligned}
$$

Hence, we get the conclusion of this lemma.

Now, we are in a position to prove the global asymptotic stability of the endemic equilibrium $\tilde{E}_{*}$ for $R_{0}>1$ by applying techniques in McCluskey [13, Proof of Theorem 4.1].

Theorem 5.1 If $R_{0}>1$ and the conditions (I) and (II) hold, then the endemic equilibrium $\tilde{E}_{*}$ of system (5.1) is globally asymptotically stable.

Proof. Let us consider the following Lyapunov functional (see McCluskey [13, Proof of Theorem 4.1]):

$$
U(t)=\frac{1}{\beta G\left(I^{*}\right)} U_{S}(t)+\frac{I^{*}}{\beta S^{*} G\left(I^{*}\right)} U_{I}(t)+U_{+}(t),
$$

where

$U_{S}(t)=g\left(\frac{S(t)}{S^{*}}\right), U_{I}(t)=g\left(\frac{I(t)}{I^{*}}\right), U_{+}(t)=\int_{0}^{h} f(\tau) \int_{t-\tau}^{t} g\left(\frac{G(I(s))}{G\left(I^{*}\right)}\right) \mathrm{d} s \mathrm{~d} \tau$. 
We now show that $\frac{\mathrm{d} U(t)}{\mathrm{d} t} \leq 0$. First, we calculate $\frac{\mathrm{d} U_{S}(t)}{\mathrm{d} t}$.

$\frac{\mathrm{d} U_{S}(t)}{\mathrm{d} t}=\frac{S(t)-S^{*}}{S^{*} S(t)}\left\{\frac{B(\mu+\delta)}{\mu}-(\mu+\delta) S(t)-\beta S(t) \int_{0}^{h} f(\tau) G(I(t-\tau)) \mathrm{d} \tau-\delta I(t)\right\}$.

By the relation $\frac{B(\mu+\delta)}{\mu}=(\mu+\delta) S^{*}+\beta S^{*} G\left(I^{*}\right)+\delta I^{*}$, we have

$$
\begin{aligned}
& \frac{\mathrm{d} U_{S}(t)}{\mathrm{d} t} \\
& =\frac{S(t)-S^{*}}{S^{*} S(t)}\left\{(\mu+\delta) S^{*}+\beta S^{*} G\left(I^{*}\right)+\delta I^{*}\right. \\
& \left.-(\mu+\delta) S(t)-\beta S(t) \int_{0}^{h} f(\tau) G(I(t-\tau)) \mathrm{d} \tau-\delta I(t)\right\} \\
& =\frac{S(t)-S^{*}}{S^{*} S(t)}\left[-(\mu+\delta)\left(S(t)-S^{*}\right)\right. \\
& \left.+\beta \int_{0}^{h} f(\tau)\left\{S^{*} G\left(I^{*}\right)-S(t) G(I(t-\tau))\right\} \mathrm{d} \tau-\delta\left(I(t)-I^{*}\right)\right] \\
& =-\frac{(\mu+\delta)\left(S(t)-S^{*}\right)^{2}}{S^{*} S(t)}+\beta G\left(I^{*}\right) \int_{0}^{h} f(\tau)\left(1-\frac{S^{*}}{S(t)}\right)\left(1-\frac{S(t)}{S^{*}} \frac{G(I(t-\tau))}{G\left(I^{*}\right)}\right) \mathrm{d} \tau \\
& -\frac{\delta\left(S(t)-S^{*}\right)\left(I(t)-I^{*}\right)}{S^{*} S(t)} \\
& =-\frac{S^{*}(\mu+\delta)\left(x_{t}-1\right)^{2}}{S(t)}+\beta G\left(I^{*}\right) \int_{0}^{h} f(\tau)\left(1-\frac{1}{x_{t}}\right)\left(1-x_{t} \tilde{y}_{t-\tau}\right) \mathrm{d} \tau \\
& -\frac{\delta I^{*}}{S(t)}\left(x_{t}-1\right)\left(y_{t}-1\right) \\
& =-\frac{S^{*}(\mu+\delta)\left(x_{t}-1\right)^{2}}{S(t)}+\beta G\left(I^{*}\right) \int_{0}^{h} f(\tau)\left(1-\frac{1}{x_{t}}-x_{t} \tilde{y}_{t-\tau}+\tilde{y}_{t-\tau}\right) \mathrm{d} \tau \\
& -\frac{\delta I^{*}}{S(t)}\left(x_{t}-1\right)\left(y_{t}-1\right) \text {. }
\end{aligned}
$$

We secondly calculate $\frac{\mathrm{d} U_{I}(t)}{\mathrm{d} t}$.

$$
\frac{\mathrm{d} U_{I}(t)}{\mathrm{d} t}=\frac{I(t)-I^{*}}{I^{*} I(t)}\left\{\beta S(t) \int_{0}^{h} f(\tau) G(I(t-\tau)) \mathrm{d} \tau-(\mu+\gamma) I(t)\right\}
$$


By the relation $(\mu+\gamma) I^{*}=\beta S^{*} G\left(I^{*}\right)$, we have

$$
\begin{aligned}
\frac{\mathrm{d} U_{I}(t)}{\mathrm{d} t} & =\frac{I(t)-I^{*}}{I^{*} I(t)}\left(\beta S(t) \int_{0}^{h} f(\tau) G(I(t-\tau)) \mathrm{d} \tau-\beta S^{*} \frac{G\left(I^{*}\right)}{I^{*}} I(t)\right) \\
& =\beta S^{*} \frac{G\left(I^{*}\right)}{I^{*}} \int_{0}^{h} f(\tau)\left(1-\frac{I^{*}}{I(t)}\right)\left(\frac{S(t)}{S^{*}} \frac{G(I(t-\tau))}{G\left(I^{*}\right)}-\frac{I(t)}{I^{*}}\right) \mathrm{d} \tau \\
& =\beta S^{*} \frac{G\left(I^{*}\right)}{I^{*}} \int_{0}^{h} f(\tau)\left(1-\frac{1}{y_{t}}\right)\left(x_{t} \tilde{y}_{t-\tau}-y_{t}\right) \mathrm{d} \tau \\
& =\beta S^{*} \frac{G\left(I^{*}\right)}{I^{*}} \int_{0}^{h} f(\tau)\left(x_{t} \tilde{y}_{t-\tau}-\frac{x_{t} \tilde{y}_{t-\tau}}{y_{t}}-y_{t}+1\right) \mathrm{d} \tau
\end{aligned}
$$

Finally, calculating $\frac{\mathrm{d} U_{+}(t)}{\mathrm{d} t}$ gives as follows.

$$
\begin{aligned}
\frac{\mathrm{d} U_{+}(t)}{\mathrm{d} t} & =\int_{0}^{h} f(\tau)\left(g\left(\frac{G(I(t))}{G\left(I^{*}\right)}\right)-g\left(\frac{G(I(t-\tau))}{G\left(I^{*}\right)}\right)\right) \mathrm{d} \tau \\
& =\int_{0}^{h} f(\tau)\left(g\left(\tilde{y}_{t}\right)-g\left(\tilde{y}_{t-\tau}\right)\right) \mathrm{d} \tau \\
& =\int_{0}^{h} f(\tau)\left(\tilde{y}_{t}-\ln \tilde{y}_{t}-\tilde{y}_{t-\tau}+\ln \tilde{y}_{t-\tau}\right) \mathrm{d} \tau .
\end{aligned}
$$

Combining (5.3), (5.7), (5.8) and (5.9), it follows from Lemma 5.1 that

$$
\begin{aligned}
\frac{\mathrm{d} U(t)}{\mathrm{d} t}= & \frac{1}{\beta G\left(I^{*}\right)}\left\{-\frac{S^{*}(\mu+\delta)\left(x_{t}-1\right)^{2}}{S(t)}\right. \\
& \left.+\beta G\left(I^{*}\right) \int_{0}^{h} f(\tau)\left(1-\frac{1}{x_{t}}-x_{t} \tilde{y}_{t-\tau}+\tilde{y}_{t-\tau}\right) \mathrm{d} \tau-\frac{\delta I^{*}}{S(t)}\left(x_{t}-1\right)\left(y_{t}-1\right)\right\} \\
& +\frac{I^{*}}{\beta S^{*} G\left(I^{*}\right)}\left\{\beta S^{*} \frac{G\left(I^{*}\right)}{I^{*}} \int_{0}^{h} f(\tau)\left(x_{t} \tilde{y}_{t-\tau}-\frac{x_{t} \tilde{y}_{t-\tau}}{y_{t}}-y_{t}+1\right) \mathrm{d} \tau\right\} \\
& +\int_{0}^{h} f(\tau)\left(\tilde{y}_{t}-\ln \tilde{y}_{t}-\tilde{y}_{t-\tau}+\ln \tilde{y}_{t-\tau}\right) \mathrm{d} \tau \\
= & -\frac{S^{*}(\mu+\delta)\left(x_{t}-1\right)^{2}}{\beta G\left(I^{*}\right) S(t)}-\frac{\delta I^{*}}{\beta G\left(I^{*}\right) S(t)}\left(x_{t}-1\right)\left(y_{t}-1\right)-\left(g\left(y_{t}\right)-g\left(\tilde{y}_{t}\right)\right) \\
& -\int_{0}^{h} f(\tau)\left\{g\left(\frac{1}{x_{t}}\right)+g\left(\frac{x_{t} \tilde{y}_{t-\tau}}{y_{t}}\right)\right\} \mathrm{d} \tau \\
\leq & -\frac{S^{*}(\mu+\delta)\left(x_{t}-1\right)^{2}+\delta I^{*}\left(x_{t}-1\right)\left(y_{t}-1\right)+\beta G\left(I^{*}\right) S(t) C_{1} C_{2} I^{*}\left(y_{t}-1\right)^{2}}{\beta G\left(I^{*}\right) S(t)} .
\end{aligned}
$$

By Lemma 4.2, for any $0<\varepsilon<v_{1}$, there exists a $T_{\varepsilon}>h$ such that $S(t)>v_{1}-\varepsilon$ for any $t>T_{\varepsilon}$. From the condition (II), we may restrict this $\varepsilon>0$ sufficiently small such that

$$
\delta^{2}-4 C_{1} C_{2}(\mu+\gamma)(\mu+\delta)\left(v_{1}-\varepsilon\right)<0
$$


Then, for all $t>T_{\varepsilon}$, it follows that

$$
\begin{aligned}
& \left(\delta I^{*}\right)^{2}-4 C_{1} C_{2} \beta S^{*} G\left(I^{*}\right)(\mu+\delta) I^{*} S(t) \\
= & \left(I^{*}\right)^{2}\left\{\delta^{2}-4 C_{1} C_{2}(\mu+\gamma)(\mu+\delta) S(t)\right\} \\
< & \left(I^{*}\right)^{2}\left\{\delta^{2}-4 C_{1} C_{2}(\mu+\gamma)(\mu+\delta)\left(v_{1}-\varepsilon\right)\right\}<0,
\end{aligned}
$$

from which we obtain that $\frac{\mathrm{d} U(t)}{\mathrm{d} t} \leq 0$ for all $t>T_{\varepsilon}$. We recall that by Lemma $5.1, \frac{\mathrm{d} U(t)}{\mathrm{d} t}=0$ if $x_{t}=1$ and $y_{t}=1$, or equivalently, if $S(t)=S^{*}$ and $I(t)=I^{*}$ for all $t>T_{\varepsilon}$. It follows from Lemma 2.1, 4.2 and LaSalle's invariant principle that $\tilde{E}_{*}$ of system (5.1) is globally asymptotically stable. This completes the proof.

Proof of Theorem 1.2. Summarizing results of Lemmas 2.1, 4.1, 4.2 and Theorem 5.1, we obtain the conclusion of this theorem.

\section{Applications}

In this section, we illustrate some examples in order to validate the feasibility of our analytical results for $R_{0}>1$ for the following SIRS epidemic model proposed in $\mathrm{Xu}$ and $\mathrm{Ma}[18]$ :

$$
\left\{\begin{array}{l}
\frac{\mathrm{d} S(t)}{\mathrm{d} t}=B-\mu S(t)-\beta S(t) G(I(t-h))+\delta R(t) \\
\frac{\mathrm{d} I(t)}{\mathrm{d} t}=\beta S(t) G(I(t-h))-(\mu+\gamma) I(t) \\
\frac{\mathrm{d} R(t)}{\mathrm{d} t}=\gamma I(t)-(\mu+\delta) R(t), h \geq 0
\end{array}\right.
$$

where $G(I)=\frac{I}{1+\alpha I}, \alpha>0$.

If $R_{0}<1$, then the disease-free equilibrium $E_{0}$ of system (6.1) is globally asymptotically stable. If $R_{0}>1$, then system (6.1) is permanent. Concerning the global stability of a unique endemic equilibrium $E_{*}$ for $R_{0}>1$, Muroya et al. [17, Corollary 4.1] established the following theorem:

Theorem A If $R_{0}>1$ and

$$
\delta>\bar{\delta}_{1}(\alpha):=\frac{\beta \gamma}{\alpha(\mu+\gamma)+\beta}-\mu,
$$

then the endemic equilibrium $E_{*}$ of system (6.1) is globally asymptotically stable.

Their result for $p=1$ improved the global stability condition of the endemic equilibrium in $\mathrm{Xu}$ and $\mathrm{Ma}$ [18, Theorem 3.1]. On the other hand, for $R_{0}>1$, similar to Theorem 1.2, we establish the following result: 


\begin{tabular}{cccc}
\hline Parameter & Description & Value & Reference \\
\hline \hline$\beta$ & Transmission rate & 0.05 per day per inidividual & Assumed \\
$B$ & Recruitment rate & 30 individuals per day & Assumed \\
$\mu$ & Natural death rate & 0.02 per day & {$[4]$} \\
$\gamma$ & Recovery rate of infectives & 0.077 per day & {$[4]$} \\
$h$ & Latency period & 0.1 days & Assumed \\
\hline
\end{tabular}

Table 1: Parameters of system (6.1) and their values used in Figure 2. For the above parameter values, we have $R_{0}=257.732 \cdots>1$.

Corollary 6.1 If $R_{0}>1$ and

$$
0 \leq \delta<\underline{\delta}_{2}(\alpha)
$$

where

$\underline{\delta}_{2}(\alpha):=\frac{2 C_{1} C_{2}(\mu+\gamma) B}{\mu+\beta G(B / \mu)}+\sqrt{\left\{\frac{2 C_{1} C_{2}(\mu+\gamma) B}{\mu+\beta G(B / \mu)}\right\}^{2}+\frac{4 \mu C_{1} C_{2}(\mu+\gamma) B}{\mu+\beta G(B / \mu)}}$,

then the endemic equilibrium $E_{*}$ of system (6.1) is globally asymptotically stable.

Next, for system (6.1), using parameter values given in Table 1, we carry out some computational experiments to investigate the feasibility of our global stability condition (6.3) with respect to the rate of immunity loss $\delta \geq 0$. For the parameter values, we obtain $R_{0}=257.732 \cdots>1$ and the endemic equilibrium $E_{*}$ exists. In Figure 1, we show regions of global and local stability of the endemic equilibrium $E_{*}$, which are obtained by Theorem A, Corollary 6.1 and $\mathrm{Xu}$ and Ma $[18$, Section 2] in the parameter space $(\alpha, \delta)$.

First, we consider the case $\alpha=1.4$. Then we obtain that $\bar{\delta}_{1}(\alpha)=0.0007 \cdots$ by (6.2) and $\underline{\delta}_{2}(\alpha)=0.0021 \cdots$ by (6.3). Hence, the endemic equilibrium $E_{*}$ of system (6.1) is globally asymptotically stable for any $\delta \geq 0$. From a biological point of view, the prevalence of the disease settles to an endemic steady state independently of initial conditions concerning the fractions of a host population for any rate of immunity loss.

Second, we consider the case $\alpha=1.1$. Then we obtain $\bar{\delta}_{1}(\alpha)=0.0045 \cdots$ by (6.2) and $\underline{\delta}_{2}(\alpha)=0.0021 \cdots$ by (6.3). Therefore, the endemic equilibrium $E_{*}$ of system (6.1) is globally asymptotically stable for $0 \leq \delta<0.0021 \cdots$ or $\delta>0.0045 \cdots$. Thus, the global stability of the endemic equilibrium $E_{*}$ is guaranteed for a small rate of immunity $\operatorname{loss} \delta$ even if the condition (6.2) in Theorem A does not hold.

On the other hand, Figure 2 indicates that the endemic equilibrium $E_{*}$ of system (6.1) is also globally asymptotically stable for the case $\alpha=1.1$ and $\delta=0.003 \in\left[\underline{\delta}_{2}(\alpha), \bar{\delta}_{1}(\alpha)\right]$ with the parameter values in Table 1 . There is still an open problem to determine the global asymptotic stability of the endemic equilibrium $E_{*}$ of system (6.1) when both of the conditions (6.2) and (6.3) fail. 


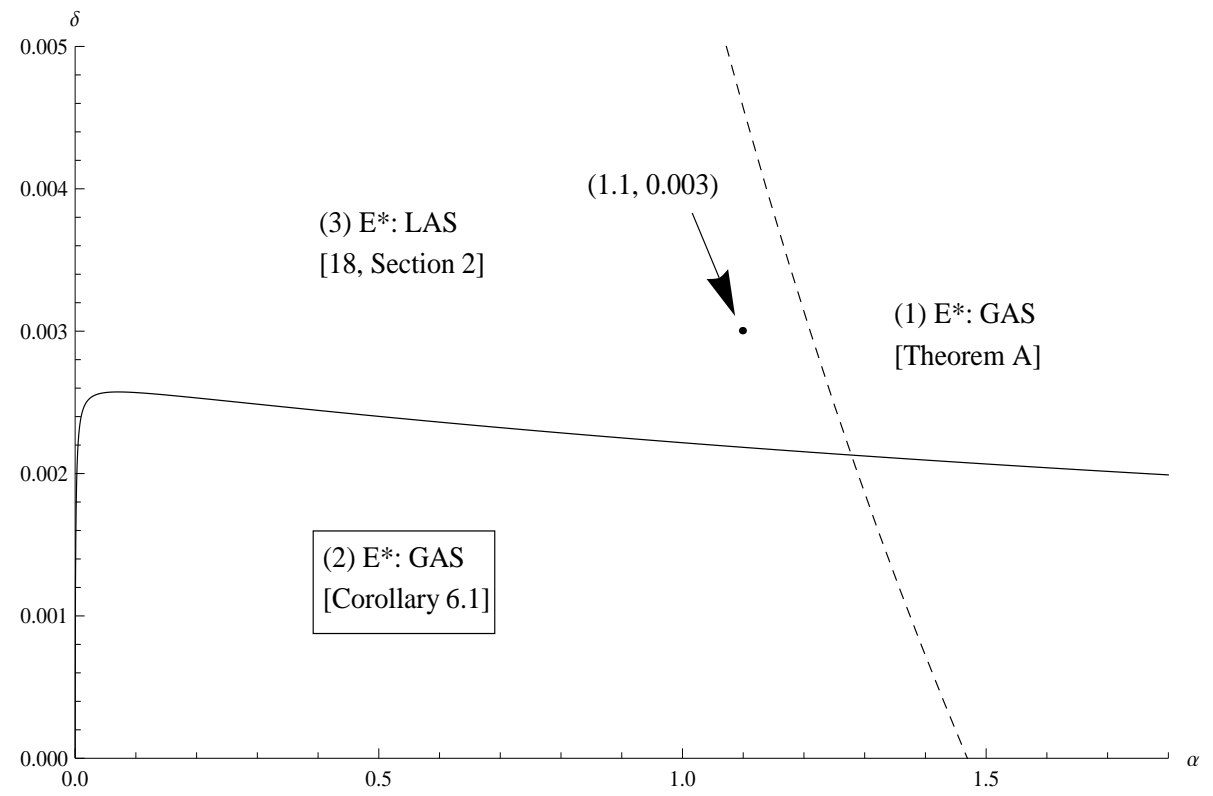

Figure 1: Curves of $\bar{\delta}_{1}(\alpha)$ (dotted line) and $\underline{\delta}_{2}(\alpha)$ (dashed line) for the parameter set in Table $1\left[(1): \delta>\bar{\delta}_{1}(\alpha),(2): 0 \leq \delta \leq \bar{\delta}_{1}(\alpha)\right.$ and $0 \leq \delta<\underline{\delta}_{2}(\alpha),(3): 0 \leq \delta \leq \bar{\delta}_{1}(\alpha)$ and $\left.\delta \geq \underline{\delta}_{2}(\alpha)\right]$. Here, GAS and LAS denote globally asymptotically stable and locally asymptotically stable, respectively.

\section{Discussion}

In this paper, we consider delayed SIRS epidemic models with a class of nonlinear incidence rates. For the nonlinear incidence rate, we put the hypotheses (H1) and (H2), which describe the crowding (saturation) effects observed in the literature of epidemiology. For $R_{0}<1$, we establish the global asymptotic stability of the disease-free equilibrium in Theorem 1.1 and for $R_{0}>1$, we obtain sufficient conditions of the global asymptotic stability of the endemic equilibrium in Theorem 1.2.

In particular, for $R_{0}>1$, by using strict monotonicity of the functions $G(I)$ and $I / G(I)$ on a neighborhood of $I^{*}$, we establish Lemma 5.1, which plays a key role to construct a Lyapunov functional for the reduced limit system (5.1).

For the special case $\delta=0$ (a delayed SIR epidemic model), Beretta and Takeuchi [1] obtained the global stability of a disease-free equilibrium and the local stability of an endemic equilibrium of the model with a bilinear incidence rate (i.e. $G(I)=I$ ). However, on their global stability analysis of the endemic equilibrium, they required a condition that the size of time delay $h$ should be small enough. The global stability of the endemic equilibrium for a sufficiently large $h$ remained unsolved for a long time. Later, by applying techniques of equation deformation on a calculation of the time derivative of a Lyapunov 


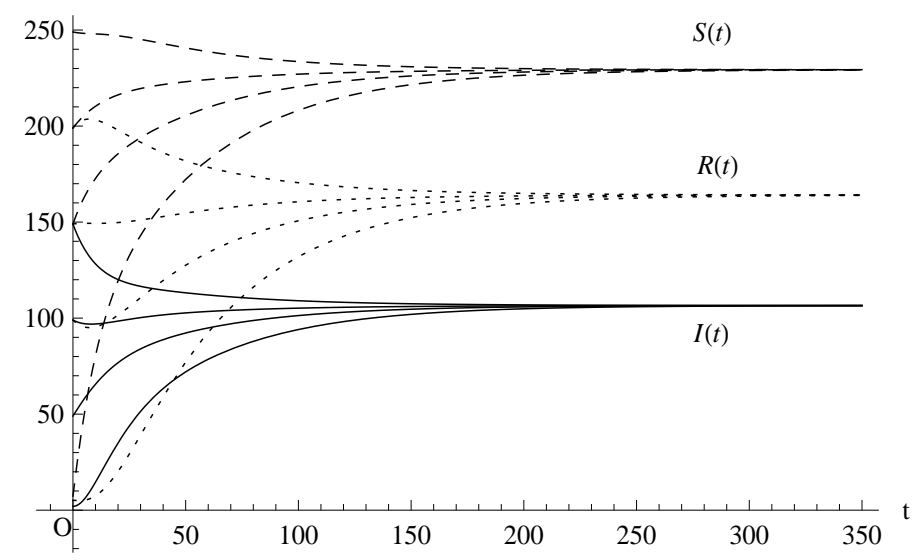

Figure 2: The graph trajectory of $S(t), I(t)$ and $R(t)$ of system (6.1). For the parameter values in Table 1 with $\alpha=1.1$ and $\delta=0.003$, we have $R_{0}=257.732 \cdots>1$ and $E^{*}=$ $(229.338 \cdots, 106.56 \cdots, 164.102 \cdots)$.

functional, McCluskey [13] solved the problem and established that the endemic equilibrium of the model is globally asymptotically stable whenever it exists. The similar global stability results for delayed SIR epidemic models with a wide class of incidence rates are now obtained in $[3,6,14]$.

On the other hand, for the case $\delta>0$, there are few global stability results concerning an endemic equilibrium of delayed SIRS epidemic models. For a delayed SIRS epidemic model with the saturated incidence rate, by applying new monotone iterative techniques in [16], Muroya et al. [17] recently obtained sufficient conditions which ensure the global asymptotic stability of an endemic equilibrium of the model with an incidence rate $\beta S(t) \frac{I(t-h)}{1+\alpha I^{p}(t-h)}$ with $p>0$ for large $\delta$. Their result for $p=1$ improved the global stability condition of the endemic equilibrium in $\mathrm{Xu}$ and $\mathrm{Ma}[18]$ for (6.1) (see Theorem A). For the system (6.1), we derive Corollary 6.1 from Theorem 1.2 and find the new global stability region of the endemic equilibrium in the parameter space $(\alpha, \delta)$, compared with that of Theorem A (see Figure 1). This illustrates that the global stability of the endemic equilibrium still holds for small $\delta$ as well as the case $\delta=0$.

\section{Acknowledgements}

The authors wish to express their gratitude to the editor and an anonymous referee for very helpful comments and valuable suggestion which improved the quality of this paper. The authors' work was supported in part by JSPS Fellows, No.237213 of Japan Society for the Promotion of Science to the first author, by the Grant MTM2010-18318 of the MICINN, Spanish Ministry of Science and 
Innovation to the second author, and by Scientific Research (c), No.21540230 of Japan Society for the Promotion of Science to the third author.

\section{References}

[1] E. Beretta and Y. Takeuchi, Convergence results in SIR epidemic models with varying population size, Nonlinear Anal. 28 (1997) 1909-1921.

[2] V. Capasso and G. Serio, A generalization of the Kermack-McKendrick deterministic epidemic model, Math. Biosci. 42 (1978) 43-61.

[3] Y. Enatsu, Y. Nakata and Y. Muroya, Global stability of SIR epidemic models with a wide class of nonlinear incidence rates and distributed delays, Discrete Continuous Dynamical System, Series B. 15 (2011) 61-74.

[4] K. Glass, Y. Xia and B.T. Grenfell, Interpreting time-series analyses for continuous-time biological models - measles as a case study, J. Theor. Biol. 2 (2003) 19-25.

[5] J. Hale, Theory of Functional Differential Equations, Springer-Verlag, Heidelberg, 1977.

[6] G. Huang, Y. Takeuchi, W. Ma and D. Wei, Global stability for delay SIR and SEIR epidemic models with nonlinear incidence rate, Bull. Math. Biol. 72 (2010) 1192-1207.

[7] H.F. Huo and Z.P. Ma, Dynamics of a delayed epidemic model with nonmonotonic incidence rate, Commun. Nonlinear Sci. Numer. Simulat. 15 (2010) 459-468.

[8] Y. Jin, W. Wang and S. Xiao, An SIRS model with a nonlinear incidence rate, Chaos, Solitons and Fractals 34 (2007) 1482-1497.

[9] A. Korobeinikov, P.K. Maini, Nonlinear incidence and stability of infectious disease models, Math. Med. Biol. 22 (2005) 113-128.

[10] A. Korobeinikov, Lyapunov functions and global stability for SIR and SIRS epidemiological models with non-linear transmission, Bull. Math. Biol. 68 (2006) 615-626.

[11] A. Korobeinikov, Global Properties of Infectious Disease Models with Nonlinear Incidence, Bull. Math. Biol. 69 (2007) 1871-1886.

[12] W.M. Liu, S.A. Levin and Y. Iwasa, Influence of nonlinear incidence rates upon the behavior of SIRS epidemiological models. J. Math. Biol. 23 (1986) $187-204$.

[13] C.C. McCluskey, Complete global stability for an SIR epidemic model with delay-Distributed or discrete, Nonl. Anal. RWA. 11 (2010) 55-59. 
[14] C.C. McCluskey, Global stability of an SIR epidemic model with delay and general nonlinear incidence, Math. Biosci. Engi. 7 (2010) 837-850.

[15] J. Mena-Lorca and H.W. Hethcote, Dynamic models of infectious diseases as regulators of population size, J. Math. Biol. 30 (1992) 693-716.

[16] Y. Muroya, Y. Enatsu and Y. Nakata, Global stability of a delayed SIRS epidemic model with a non-monotonic incidence rate, J. Math. Anal. Appl. 377 (2011) 1-14.

[17] Y. Muroya, Y. Enatsu and Y. Nakata, Monotone iterative techniques to SIRS epidemic models with nonlinear incidence rates and distributed delays, Nonl. Anal. RWA. 12 (2011) 1897-1910.

[18] R. Xu and Z. Ma, Stability of a delayed SIRS epidemic model with a nonlinear incidence rate, Chaos, Solitons and Fractals 41 (2009) 2319-2325.

[19] Y. Yang and D. Xiao, Influence of latent period and nonlinear incidence rate on the dynamics of SIRS epidemiological models, Discrete and Continuous Dynamical Systems Series B 13 (2010) 195-211. 